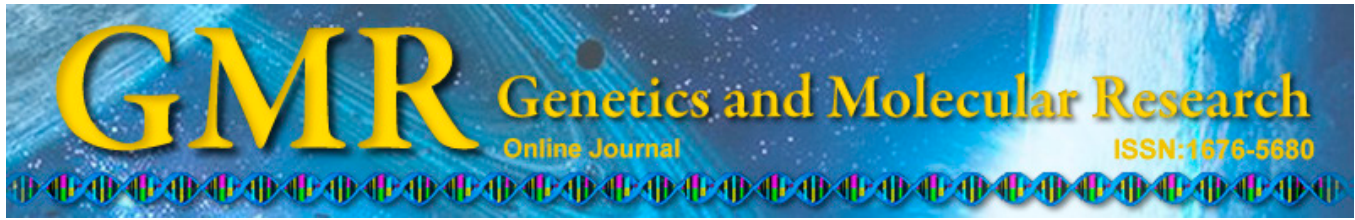

\title{
Establishment and rapid detection of a heterozygous missense mutation in the CACNA1F gene by ARMS technique with double-base mismatched primers
}

\author{
W.C. Yang ${ }^{1 *}$, L. Zhu ${ }^{1 *}$, B.X. Zhou ${ }^{1 *}$, S. Tania ${ }^{1}$, Q. Zhou ${ }^{2}$, M.A. Khan ${ }^{1}$, \\ X.L. Fu' ${ }^{1}$, J.L. Cheng', H.B. Lv $^{2}$ and J.J. Fu ${ }^{1}$ \\ ${ }^{1}$ Key Laboratory of Epigenetics and Oncology, \\ The Research Center for Preclinical Medicine, Luzhou Medical College, \\ Luzhou, Sichuan, China \\ ${ }^{2}$ Department of Ophthalmology, Affiliated Hospital of Luzhou Medical College, \\ Luzhou, Sichuan, China \\ *These authors contributed equally to this study. \\ Corresponding authors: H.B. Lv / J.J. Fu \\ E-mail: oculistlvhongbin@163.com / fujunjiang@hotmail.com \\ Genet. Mol. Res. 14 (3): 11480-11487 (2015) \\ Received February 6, 2015 \\ Accepted June 26, 2015 \\ Published September 25, 2015 \\ DOI http://dx.doi.org/10.4238/2015.September.25.14
}

\begin{abstract}
Retinitis pigmentosa (RP) is a retinal degenerative disorder that often causes complete blindness. Mutations of more than 50 genes have been identified as associated with RP, including the $C A C N A 1 F$ gene. In a recent study, by employing next-generation sequencing, we identified a novel mutation in the CACNAIF gene. In this study, we used the amplification refractory mutation system (ARMS) and identified a single nucleotide change c.1555C $>\mathrm{T}$ in exon 13 of the $C A C N A 1 F$ gene, leading to the substitution of arginine by tryptophan (p.R519W) in a Chinese individual affected by RP.
\end{abstract}


This study actually confirms this novel mutation, and establishes the ARMS technique for the detection of mutations in RP.

Key words: $C A C N A 1 F$; Missense mutation; Retinitis pigmentosa; Amplification refractory mutation system; Double-base mismatch

\section{INTRODUCTION}

Retinitis pigmentosa (RP; Online Mendelian Inheritance in Man No. 268000) comprises a heterogeneous group of hereditary retinal degenerative disorders that cause progressive loss of photoreceptors, severe vision impairment, night blindness, abnormal color vision, fundus degeneration, and often complete blindness (Hartong et al., 2009; Wu et al., 2014). RP affects approximately 1 in 4000 people and more than 1 million individuals worldwide (Katagiri et al., 2014). According to the RetNet database, mutations in more than 50 genes are associated with RP (Wang et al., 2014). Different mutations in different genes can have similar consequences. However, different mutations in the same gene may cause different diseases/ phenotypes (Yang et al., 2014).

The $C A C N A 1 F$ gene (Online Mendelian Inheritance in Man No. 300110) encodes a voltage-dependent L-type calcium channel, the $\mathrm{Ca}_{\mathrm{v}} 1.4$ channel. It is expressed in both rod and cone terminals mediating neurotransmitter secretion at ribbon synapses of the retinal photoreceptors, and therefore plays an important role in signal transmission from photoreceptors to bipolar cells in the retinal neurons (Baumann et al., 2004; Hauke et al., 2013). Mutations in the $C A C N A 1 F$ gene are associated with some progressive retinal disorder with a large phenotypic variability (Hope et al., 2005). A recent study that used next-generation sequencing reported a missense mutation in the CACNA1F gene associated with RP (Zhou et al., 2015).

Various molecular techniques have been developed to detect mutations and, until recently, Sanger sequencing was the main method for RP molecular diagnosis. However, it appears that this technique is not ideal for the rapid screening of large numbers of samples, and is expensive in terms of reagents and labor; next-generation sequencing might provide a solution (Fu et al., 2013b). However, next-generation sequencing is a sophisticated tool and is also expensive. The amplification refractory mutation system (ARMS) may provide a promising alternative for molecular diagnosis because it is rapid, simple, cost-effective, and safe for the detection of known point mutations worldwide (Hassan et al., 2013; Hanafi et al., 2014). Newton et al. (1989) first described the ARMS technique, which is a polymerase chain reaction (PCR)-based method, and requires only targeted PCR amplification and gel electrophoresis of the amplicon. In this method, an allele-specific oligonucleotide primer with a triple end is used with a common primer in one PCR in which the presence of an amplified mutant detected by agarose gel electrophoresis indicates the presence of the mutation allele. Absence of the amplified mutant suggests the presence of the normal DNA sequence at that specific site. In parallel, a corresponding normal primer is used with a common primer in another PCR in which the presence of a normal amplified product suggests presence of a normal DNA sequence at that specific site, while its absence suggests the presence of the mutant allele (Newton et al., 1989). In this study, we used the ARMS technique to establish and detect a missense mutation in the CACNA1F gene associated with RP. 


\section{MATERIAL AND METHODS}

\section{Clinical diagnosis and sample collection}

The Chinese proband suffering from RP in this study has been described previously (Zhou et al., 2015). Genomic DNA from the proband and his family was isolated from peripheral leukocytes using a previously described method (Fu et al., 2000, 2002; Fu, 2012; Zhou et al., 2015).

\section{Design of ARMS primers}

ARMS primers for the M067 family with wild type and mutant were designed at the 5'end with one base mismatched or two bases mismatched, as shown in Table 1, according to the heterozygous missense mutation (c.1555C > T, p.R519W) located in exon 13 of the CACNA1F gene (GenBank accession No.: NM_005183, NP_005174) on the human X chromosome (Zhou et al., 2015). A common primer or ordinary primer was located at the 3 '-end of this gene.

\section{ARMS-PCR and agarose gels electrophoresis}

The PCR system ( $10 \mu \mathrm{L}$ total) comprised: $1 \mu \mathrm{L} 2.5 \mu \mathrm{M}$ primers, $1 \mu \mathrm{L}$ DNA template (10 ng), $5 \mu \mathrm{L} 2 \mathrm{X}$ PCR Taq Master Mix (TianGen Biotech Co. Ltd., Beijing, China), and $3 \mu \mathrm{L}$ double-distilled $\mathrm{H}_{2} \mathrm{O}$. The PCR regimen was as follows: initial denaturation at $95^{\circ} \mathrm{C}$ for $90 \mathrm{~s}$, followed by 32 cycles of $40 \mathrm{~s}$ at $94^{\circ} \mathrm{C}, 30 \mathrm{~s}$ at $63^{\circ} \mathrm{C}, 30 \mathrm{~s}$ at $72^{\circ} \mathrm{C}$, and a final extension of 5 min at $72^{\circ} \mathrm{C}$. The PCR amplification was executed in an Applied Biosystems Veriti ${ }^{\circledR}$ 96-Well Thermal Cycler (Life Technologies, USA) (Fu et al., 2013a). The amplified PCR products were resolved by electrophoresis on a $1 \%$ agarose gel in $1 \mathrm{X}$ TAE (Tris-acetate-ethylenediaminetetraacetic acid) buffer at $120 \mathrm{~V}$ for $30 \mathrm{~min}$. The agarose gels were then visualized by $0.5 \mu \mathrm{g} / \mathrm{mL}$ ethidium bromide staining, and the images were documented using ChemiDoc XR (Bio-Rad, USA) (Fu, 2012; Fu et al., 2013a). The DNA marker (DL2000) used in the electrophoresis was purchased from TaKaRa Biotechnology (Dalian) Co. Ltd., China.

\section{Mutation validation}

The putative mutations detected by ARMS were validated by Sanger sequencing on an ABI3500 sequencer (Applied Biosystems Inc., Foster City, CA, USA) (Zhou et al., 2015).

\section{RESULTS}

\section{ARMS-PCR results}

Wild-type and mutant ARMS primers for the M067 family were designed at the 3'end with one or two bases mismatched (Table 1). The primer pair CACNA1F-AP-RC and CACNA1F-AP-LW specific for the wild type was used for detecting the CACNA1F gene allele, the primer pair CACNA1F-AP-RC and CACNA1F-AP-LM was used for detecting the mutant $C A C N A 1 F$ gene c. $1555 \mathrm{C}>\mathrm{T}$, and the primer pair CACNA1F-AP-RC and CACNA1F-AP-LM2 with two base mismatches was used for enhanced detection of the mutant $C A C N A 1 F$ gene c. $1555 \mathrm{C}>\mathrm{T}$. 
Table 1. Amplification refractory mutation system (ARMS) primers, combinations, and amplification conditions.

\begin{tabular}{|c|c|c|c|c|c|}
\hline Primer name & Sequences $\left(5^{\prime}-3^{\prime}\right)$ & $\operatorname{Tm}\left({ }^{\circ} \mathrm{C}\right)$ & Size (bp) & Cycles & Mismatched base No. \\
\hline CACNA1F-AP-LW & CCGAGCCAACCGGGTCCTTC & 63 & 521 & 33 & 1 (comparing mutant) \\
\hline CACNA1F-AP-LW2 & CCGAGCCAACCGGGTCCATC & 63 & 521 & 33 & 2 (comparing mutant) \\
\hline CACNA1F-AP-LM & CCGAGCCAACCGGGTCCTTT & 63 & 521 & 33 & 1 (comparing wild type) \\
\hline CACNA1F-AP-LM2 & CCGAGCCAACCGGGTCCATT & 63 & 521 & 33 & 2 (comparing wild type) \\
\hline CACNA1F-AP-RC & GGCTGCCTGGGTTCTAATTCT & 63 & 521 & 33 & 0 (common primer) \\
\hline
\end{tabular}

Nucleotides in bold on the primers correspond to the introduced mismatches. The common primer was used as a 3 '-end primer.

The results of detecting mutant and wild types by ARMS-PCR are shown in Figure 1.

a

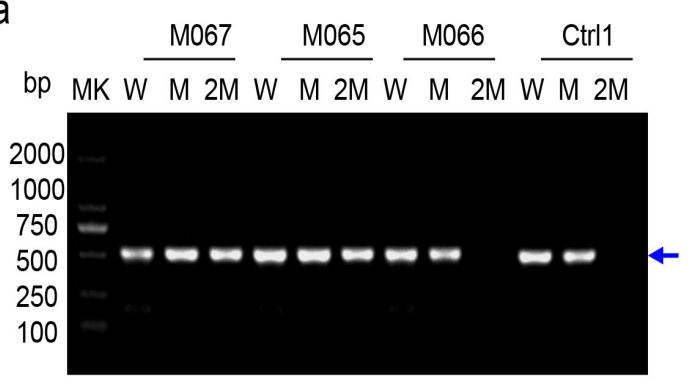

b

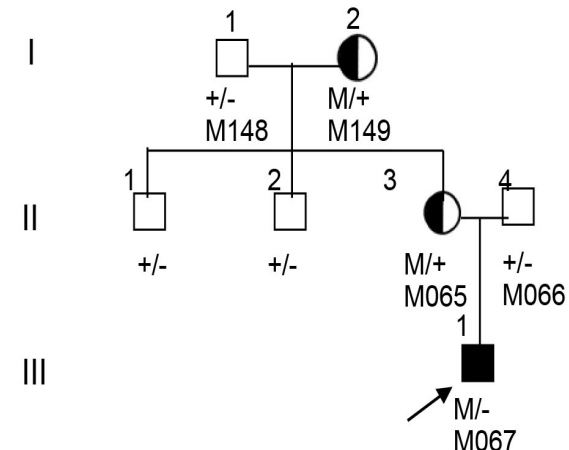

Figure 1. Establishment and detection of the $C A C N A 1 F$ gene point mutation by amplification refractory mutation system-polymerase chain reaction (ARMS-PCR). The blue arrow indicates the PCR bands in the different samples. (a) ARMS-PCR with different mismatched primers. The lane labeled " $M K$ " indicates the DNA molecular weight marker DL2000 with the indicated size (bp). Lanes labeled " $W$ " indicate ARMS-PCR using primers CACNA1FAP-RC and CACNA1F-AP-LW specific for the wild type. Lanes labeled " $M$ " indicate ARMS-PCR using primers CACNA1F-AP-RC and CACNA1F-AP-LM specific for the mutant. Lanes labeled " $2 M$ " indicate ARMS-PCR using primers CACNA1F-AP-RC and CACNA1F-AP-LM2 specific for the mutant with two mismatched base pairs. "Ctrl1" indicates normal control female DNA. (b) Pedigree and segregation of the CACNA1F mutation. Lane $M$ indicates the mutant allele of the CACNA1F gene, c.1555C>T, p.R519W; "+" indicates the c.C1555 normal allele of the $C A C N A 1 F$ gene.

From Figure 1, we can see that the primer pair CACNA1F-AP-RC and CACNA1F-AP-LM, with one base mismatch, amplified non-specific bands in all individuals (Figure 1a, lanes labeled "M"). Only the primer pair CACNA1F-AP-RC and CACNA1F-AP-LM2, with two base mismatches, amplified specific bands corresponding to the mutant allele in the male patient M067 (Figure 1a, lane labeled "2M") and the carrier M065 (Figure 1a, lane labeled " $2 \mathrm{M}$ "), whereas the normal female control and the normal male without mutation showed no amplification (Figure 1a, lanes labeled "2M"). The primer pair CACNA1F-AP-RC and CACNA1F-AP-LW specific for the wild type amplified the PCR products in the lane labeled "M" of the patient M067 (Figure 1a), which indicates that CACNA1F-AP-LW is not specific. Therefore, we designed another primer CACNA1F-AP-LW2 with a mismatch from " $T$ " to "A" at the third position from the 3'-end for detecting the wild-type allele.

Using the primer pair CACNA1F-AP-RC and CACNA1F-AP-LW2, and the primer pair CACNA1F-AP-RC and CACNA1F-AP-LM2, we found that the patient only amplified 
the mutant allele but did not amplify the wild type allele, whereas the carrier mother M065 and the grandmother M149 amplified both alleles, and normal individuals including M066 and M148 from this family, and the normal "Ctrl1", only amplified the wild-type allele (Figure 2). There was no amplification in the "Ctrl2" lane, which did not include a DNA template (Figure 2).

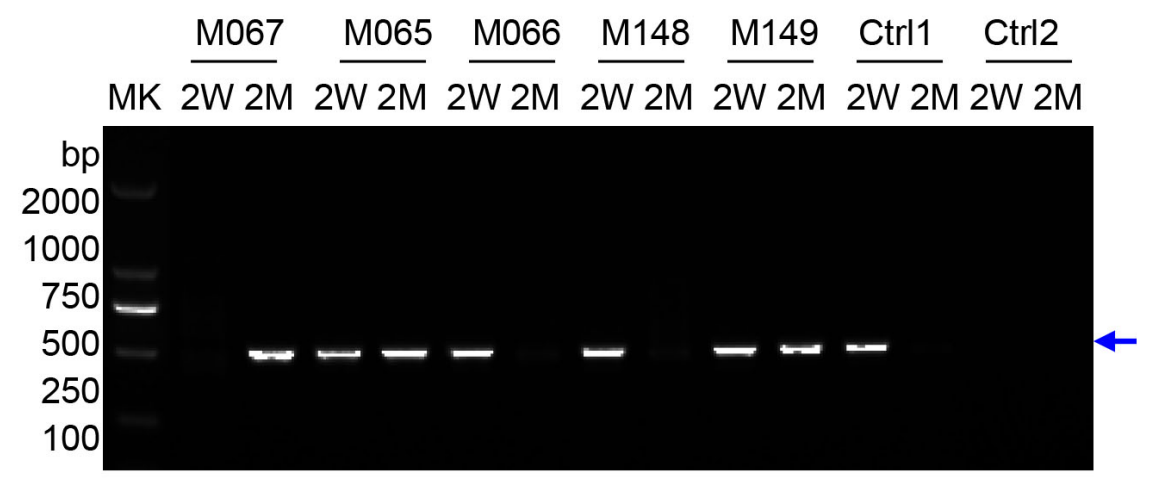

Figure 2. Successful amplification refractory mutation system-polymerase chain reaction (ARMS-PCR) detection by primers with double-mismatched base pairs. The blue arrow indicates the PCR bands in the different samples. The lane labeled "MK" indicates the DNA molecular weight marker DL2000 with the fragment size (bp). Lanes labeled " $2 W$ " indicate ARMS-PCR using primers CACNA1F-AP-RC and CACNA1F-AP-LW2 specific for the wild-type allele with one mismatched base pair. Lanes labeled " $W$ ", " $M$ ", and " $2 M$ " are described in Figure 1. "Ctrl1" indicates normal control female DNA. "Ctrl2" indicates no DNA template.

\section{Mutation validation of ARMS-PCR}

Validation of ARMS-PCR was performed by Sanger sequencing and representative results are shown in Figure 3 ( $a$ and b) (Zhou et al., 2015). The study showed that the proband's grandmother had the same mutation as her grandson, suggesting that the CACNA1F mutation was passed from the grandmother (I:2) to the mother (II:3), leading to the pathogenic mutation in the male offspring, and showed perfect co-segregation with the disease in the family (Zhou et al., 2015). The father of the proband (II:4) and other members of the family were normal, with the wild-type $C A C N A 1 F$ gene (Zhou et al., 2015).

a
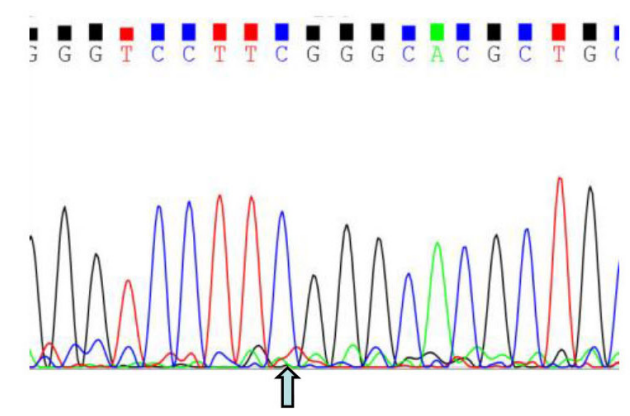

b
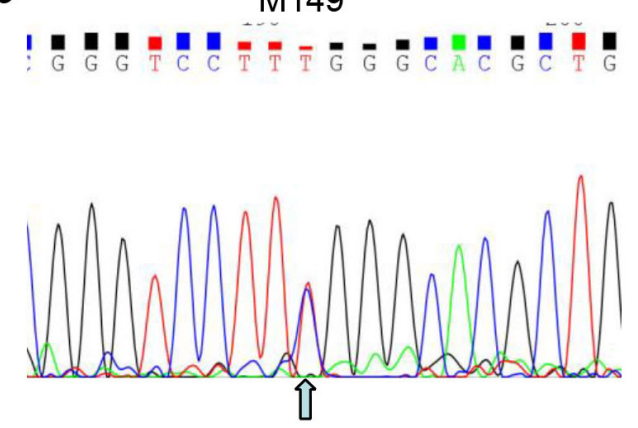

Figure 3. Sanger sequencing confirmation for the proband's grandparents. a. Grandfather M148. b. Grandmother M149. 


\section{DISCUSSION}

Molecular diagnostic techniques are a highly accurate means of helping diagnose a genetic disease. They characterize the spectrum of gene mutations, provide new information about the underlying disease-associated mutation, and may possibly lead to the development of treatments for common inherited disorders (Newton et al., 1989; Fu et al., 2013b). However, molecular diagnostic techniques have their limitations: they are time-consuming, and prohibitively expensive for laboratories with limited funds (Hassan et al., 2013). ARMS has proven to be a rapid, simple, and cost-effective technique for screening known mutations (Hanafi et al., 2014). In our study, we employed ARMS to detect a $C A C N A 1 F$ gene mutation causing RP in a Chinese family.

The $C A C N A 1 F$ gene is located on the $\mathrm{X}$ chromosome at $\mathrm{p} 11.23$. It encodes a poreforming protein of 1977 amino acids with cytoplasmic amino- and carboxyl-termini separated by four homologous repeat domains (I-IV), each consisting of six transmembrane segments (S1-S6). CACNA1F is expressed preferentially in the inner and outer nuclear layers of the retina, indicative of a specific functional role in visual processing, and mutation in this gene may cause functional abnormality associated with retinal disorders (Naylor et al., 2000).

Mutations in $C A C N A 1 F$ are mostly associated with incomplete X-linked congenital stationary night blindness type 2A (CSNB2A) (Hauke et al., 2013). According to the Human Gene Mutation Database, more than 60 different $C A C N A 1 F$ mutations have been reported to cause CSNB2A. A few mutations in the $C A C N A 1 F$ gene have also been reported to be associated with Aland Island eye disease (Jalkanen et al., 2007), X-linked cone-rod dystrophy (CORDX3) (Jalkanen et al., 2006), or other retinal disorders (Hemara-Wahanui et al., 2005; Hope et al., 2005). In 13 families with incomplete CSNB2A, Strom et al. (1998) identified nine different mutations in 10 families, including three nonsense and one frameshift mutation, and indicated that $C A C N A 1 F$ mutations cause a decrease in neurotransmitter release from photoreceptor presynaptic terminals in CSNB2A patients. Jacobi et al. (2003) identified a $\mathrm{C}$ deletion at nucleotide 4548 in $C A C N A 1 F$ in two affected members of a French family with CSNB2A, resulting in a frameshift with a predicted premature termination at codon 1524. Jalkanen et al. (2007) identified a novel 425-bp deletion mutation encompassing exon 30 and portions of adjacent introns of the $C A C N A 1 F$ gene, predicted to cause a deletion of the transmembrane domain and consequently an altered membrane topology of the encoded alpha1-subunit of the $\mathrm{Ca}_{\mathrm{v}} 1.4$ calcium channel. Jalkanen et al. (2006) identified a deletion/ insertion (IVS28-1 GCGTC-TGG) at the -1 position in the splice acceptor site of intron 28 of the $C A C N A 1 F$ gene in a large Finnish family with CORDX3, resulting in premature termination and exonic deletions of the encoded protein, $\mathrm{Ca}_{\mathrm{v}} 1.4$ alpha1 subunit. Vincent et al. (2011) identified a missense mutation in $C A C N A 1 F$ causing CSNB2A and Aland Island eye disease phenotypes in a family, which confirmed that both diseases are allelic in which other genetic or environmental modifiers might influence the expression of $C A C N A 1 F$.

Here, we have identified a single nucleotide change c.1522C $>\mathrm{T}$ in exon 13 of the $C A C N A 1 F$ gene leading to the substitution of arginine by tryptophan (p.R508W) in individuals affected by RP. By employing next-generation sequencing, our recent study identified a mutation at this position in the CACNA1F gene (Zhou et al., 2015) in a Chinese family with RP. Moreover, Zhou et al. (2015) identified the same heterozygous mutation in normal females of this family, indicating that heterozygous females were carriers for RP. This study actually confirms this novel mutation, and establishes the ARMS technique with double-base mismatched primers for the detection of mutations in RP. By using ARMS, 20 different muta- 
tions of the $\beta$-globin gene could be detected effectively in a cost-efficient manner (Hanafi et al., 2014). Hassan et al. (2013) applied a simple PCR approach involving a multiplex ARMS and one ARMS technique, consisting of $20 \beta$-globin gene mutations, and successfully detected $\beta$-thalassemia patients and carriers in Malaysia. Thong et al. (2005) used a two-step molecular diagnostic strategy consisting of the ARMS technique to identify the most common mutations, followed by other DNA-based diagnostic techniques for $\beta$-thalassemia alleles, and achieved a $100 \%$ success rate in this study. Hanafi et al. (2014) applied a multiplex ARMS technique for the detection of $\beta$-globin gene mutations and successfully identified the mutation spectrum in transfusion-dependent $\beta$-thalassemia in Malay patients. Interesting, $\mathrm{Fu}$ et al. (2000) have performed rapid prenatal gene diagnosis for $\beta$-thalassemia by ARMS. However, the ARMS technique is rarely used for RP diagnosis. This study indicates ARMS as a potential molecular tool for mutation detection in RP.

Different mutations of $C A C N A 1 F$ can lead to different phenotypes having a few or more common symptoms, suggesting the need for a thorough clinical examination with visual function tests, together with mutation analysis, for an accurate diagnosis. The ARMS technique is considered an unbiased, time-efficient method, which preferentially allows detection of known mutations in known genes, as in this study. We applied the ARMS technique using double-base mismatched primers and successfully identified the known mutation in the CACNAIF gene.

\section{Conflicts of interest}

The authors declare no conflict of interest.

\section{ACKNOWLEDGMENTS}

Research supported in part by the Teaching Reform Project of Luzhou Medical College (\#JG2014086), the Science and Technology Innovation Team of the Colleges and Universities of Sichuan Province (\#13TD0032), the Applied Basic Research Program of the Science and Technology Department of Sichuan Province (\#14JC0797), the Luzhou City Special Foundation (\#2013LZLY-J10), the Innovation Project of Luzhou Medical College, and the National Natural Science Foundation of China (\#30371493 and \#81172049).

\section{REFERENCES}

Baumann L, Gerstner A, Zong X, Biel M, et al. (2004). Functional characterization of the L-type $\mathrm{Ca}^{2+}$ channel Cav1.4alpha1 from mouse retina. Invest. Ophthalmol. Vis. Sci. 45: 708-713.

Fu JJ (2012). Short Protocols in Medical Molecular Biology. China Medical Science Press, Beijing.

Fu JJ, Li LY, Li XR and Lu GX (2000). Rapid prenatal gene diagnosis for $\beta$-thalassemia by amplification refractory mutation system (ARMS). Chin. J. Obstet. Gynecol. 35: 359-360.

Fu JJ, Li LY and Lu GX (2002). Relationship between microdeletion on Y chromosome and patients with idiopathic azoospermia and severe oligozoospermia in the Chinese. Chin. Med. J. 115: 72-75.

Fu JJ, Yang L, Khan MA and Mei Z (2013a). Genetic characterization and authentication of Lonicera japonica Thunb. by using improved RAPD analysis. Mol. Biol. Rep. 40: 5993-5999.

Fu Q, Wang F, Wang H, Xu F, et al. (2013b). Next-generation sequencing-based molecular diagnosis of a Chinese patient cohort with autosomal recessive retinitis pigmentosa. Invest. Ophthalmol. Vis. Sci. 54: 4158-4166.

Hanafi S, Hassan R, Bahar R, Abdullah WZ, et al. (2014). Multiplex amplification refractory mutation system (MARMS) for the detection of $\beta$-globin gene mutations among the transfusion-dependent $\beta$-thalassemia Malay patients in Kelantan, Northeast of Peninsular Malaysia. Am. J. Blood Res. 4: 33-40. 
Hartong DT, McGee TL, Sandberg MA, Berson EL, et al. (2009). Search for a correlation between telomere length and severity of retinitis pigmentosa due to the dominant rhodopsin Pro23His mutation. Mol. Vis. 15: 592-597.

Hassan S, Ahmad R, Zakaria Z, Zulkafli Z, et al. (2013). Detection of $\beta$-globin Gene Mutations Among $\beta$-thalassaemia Carriers and Patients in Malaysia: Application of Multiplex Amplification Refractory Mutation System-Polymerase Chain Reaction. Malays. J. Med. Sci. 20: 13-20.

Hauke J, Schild A, Neugebauer A, Lappa A, et al. (2013). A novel large in-frame deletion within the CACNA1F gene associates with a cone-rod dystrophy 3-like phenotype. PLoS One 8: e76414.

Hemara-Wahanui A, Berjukow S, Hope CI, Dearden PK, et al. (2005). A CACNA1F mutation identified in an X-linked retinal disorder shifts the voltage dependence of Cav1.4 channel activation. Proc. Natl. Acad. Sci. U S A 102: 7553-7558.

Hope CI, Sharp DM, Hemara-Wahanui A, Sissingh JI, et al. (2005). Clinical manifestations of a unique X-linked retinal disorder in a large New Zealand family with a novel mutation in CACNA1F, the gene responsible for CSNB2. Clin. Experiment. Ophthalmol. 33: 129-136.

Jacobi FK, Hamel CP, Arnaud B, Blin N, et al. (2003). A novel CACNA1F mutation in a French family with the incomplete type of X-linked congenital stationary night blindness. Am. J. Ophthalmol. 135: 733-736.

Jalkanen R, Mäntyjärvi M, Tobias R, Isosomppi J, et al. (2006). X linked cone-rod dystrophy, CORDX3, is caused by a mutation in the CACNA1F gene. J. Med. Genet. 43: 699-704.

Jalkanen R, Bech-Hansen NT, Tobias R, Sankila EM, et al. (2007). A novel CACNA1F gene mutation causes Aland Island eye disease. Invest. Ophthalmol. Vis. Sci. 48: 2498-2502.

Katagiri S, Akahori M, Sergeev Y, Yoshitake K, et al. (2014). Whole exome analysis identifies frequent CNGA1 mutations in Japanese population with autosomal recessive retinitis pigmentosa. PLoS One 9: e108721.

Naylor MJ, Rancourt DE and Bech-Hansen NT (2000). Isolation and characterization of a calcium channel gene, Cacnalf, the murine orthologue of the gene for incomplete X-linked congenital stationary night blindness. Genomics 66: 324-327.

Newton CR, Graham A, Heptinstall LE, Powell SJ, et al. (1989). Analysis of any point mutation in DNA. The amplification refractory mutation system (ARMS). Nucleic Acids Res. 17: 2503-2516.

Strom TM, Nyakatura G, Apfelstedt-Sylla E, Hellebrand H, et al. (1998). An L-type calcium-channel gene mutated in incomplete X-linked congenital stationary night blindness. Nat. Genet. 19: 260-263.

Thong MK, Tan JA, Tan KL and Yap SF (2005). Characterisation of beta-globin gene mutations in Malaysian children: a strategy for the control of beta-thalassaemia in a developing country. J. Trop. Pediatr. 51: 328-333.

Vincent A, Wright T, Day MA, Westall CA, et al. (2011). A novel p.Gly603Arg mutation in CACNA1F causes Åland island eye disease and incomplete congenital stationary night blindness phenotypes in a family. Mol. Vis. 17: 3262-3270.

Wang F, Wang H, Tuan HF, Nguyen DH, et al. (2014). Next generation sequencing-based molecular diagnosis of retinitis pigmentosa: identification of a novel genotype-phenotype correlation and clinical refinements. Hum. Genet. 133: 331-345.

Wu J, Chen L, Tam OS, Huang XF, et al. (2014). Whole exome sequencing reveals genetic predisposition in a large family with retinitis pigmentosa. Biomed. Res. Int. 2014: 302487.

Yang G, Xie S, Feng N, Yuan Z, et al. (2014). Spectrum of rhodopsin gene mutations in Chinese patients with retinitis pigmentosa. Mol. Vis. 20: 1132-1136.

Zhou Q, Cheng J, Yang W, Tania M, et al. (2015). Identification of a Novel Heterozygous Missense Mutation in the CACNAIF Gene in a Chinese Family with Retinitis Pigmentosa by Next Generation Sequencing. Biomed Res. Int. 2015: 907827. 